\title{
How to face the novel coronavirus infection during the 2019-2020 epidemic: the experience of Sichuan Provincial People's Hospital
}

\author{
Lingai Pan ${ }^{1 *} \mathbb{D}$, Li Wang $^{2,3}$ and Xiaobo Huang ${ }^{1}$
}

(C) 2020 Springer-Verlag GmbH Germany, part of Springer Nature

Up to 07:58 February 6, 2020: there have been 28,060 confirmed cases of 2019-novel coronavirus (2019-nCoV) infection in China. Since the outbreak of 2019-nCoV in Wuhan, China, now there are confirmed cases in multiple countries and there are many health care workers who have been infected while they were unprotected or improperly protected. As the $2019-\mathrm{nCoV}$ is highly contagious and the transmission dynamics is still not fully understood [1], in large hospitals, such as Sichuan provincial people's hospital which has more than 4000 beds and a large number of outpatient visits every day, it was necessary to establish a hospital-specific protocol to deal with the new coronavirus infection.

January 17, 2020: the Sichuan Provincial People's Hospital officially launched the 2019 influenza emergency response plan and established a leading group for influenza prevention and control. The president of Sichuan Provincial People's Hospital serves as the team leader, and its members include related departments such as the administrative department, the intensive care unit, the infectious disease department, the respiratory department, nurses, the nosocomial infection control department and the radiology department. Afterwards we established multiple working groups: the emergency team, the prevention and control team, the medical emergency team, the material security team, the publicity and education team and the information updating team. Since the transmission dynamics were unclear,

\footnotetext{
*Correspondence: panlingai2004@163.com

1 Department of Critical Care Medicine, Sichuan Academy of Medical Sciences and Sichuan Provincial People's Hospital, University of Electronic Science and Technology of China, Chengdu, People's Republic of China Full author information is available at the end of the article
}

establishing fever clinics, isolation wards and emergency wards were the most important measures.

January 18, 2020: nosocomial infection control experts trained on isolation rules for highly contagious diseases. The infectious disease physician, the respiratory physician and an emergency medical physician became a fever diagnosis and screening team to triage the patients who have fever. The specific flowchart is shown in Fig. 1. For highly suspected patients, the rule is to enter the isolation ward and wait for the results of the New Coronavirus nucleic acid test and of the radiology examination. If the suspected patient is confirmed, then he enters the emergency ward for treatment. During the screening process, the medical staff must wear masks, wash their hands and put on isolation clothes to make sure not to be infected by $2019-\mathrm{nCoV}$ carriers. The opening of the online clinics has reduced the number of common flu patients visiting hospitals, and consultation can be conducted at the online clinics.

January 30, 2020: the hospital suspended all nonemergency patient admissions and stopped all nonemergency surgeries. The multiple departments were merged together, and the medical staff of the merged departments were on standby at home. The hospital was assigned to participate in the prevention and treatment of the new coronavirus epidemic. The objectives are: (1) to reduce the flow of personnel and reduce the chance of infection; (2) to save medical supplies and ensure that front-line medical staff who fight the 2019-nCoV have protective supplies available.

Due to the increasing severity of the coronavirus epidemic in the country, laboratory testers are under great pressure and high risk. On January 25, 2020, the enhanced biosafety secondary laboratory project was 


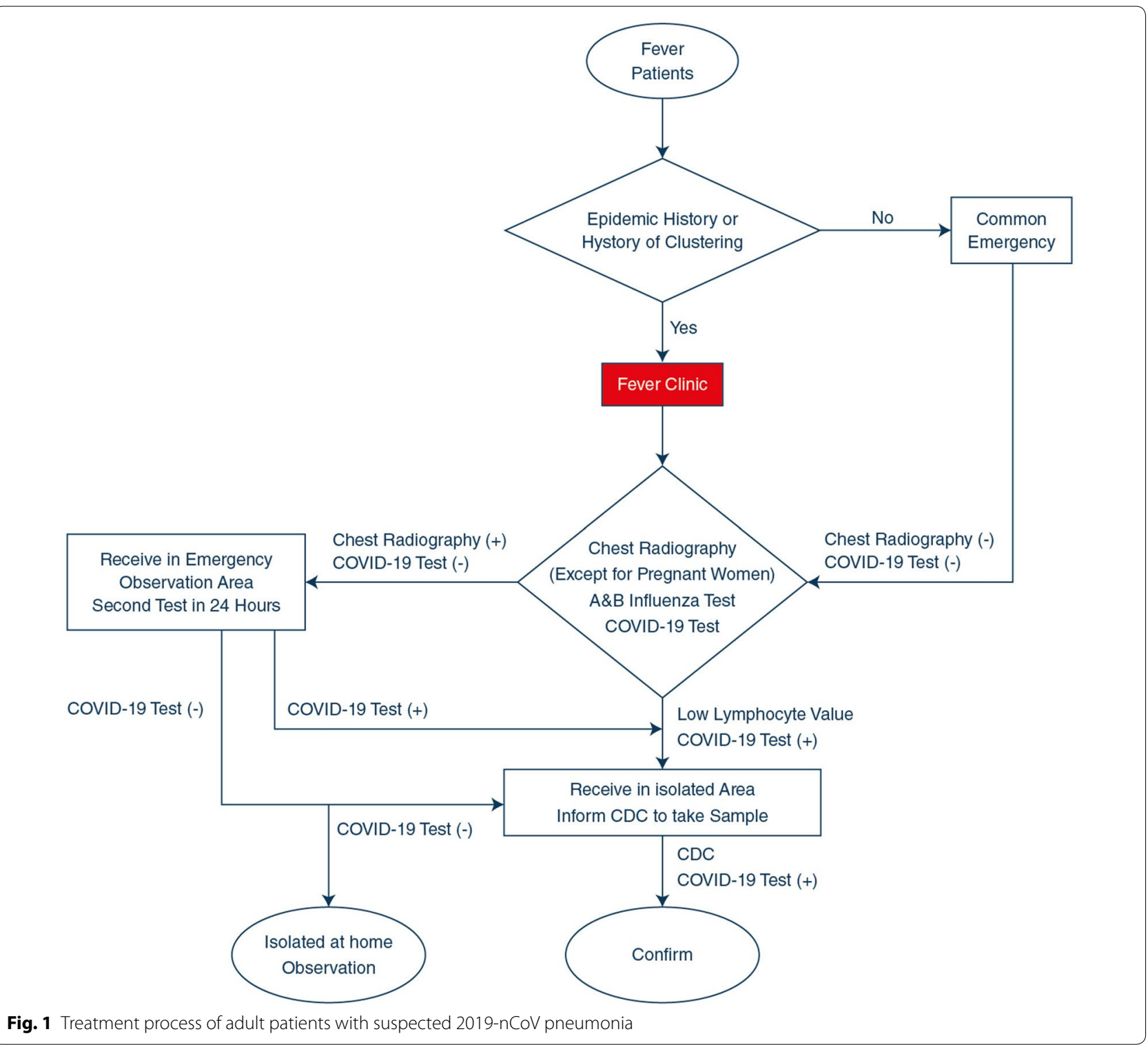

launched. It took 6 days for the construction and 2 days for the adjustment. It was officially put into use on February 4,2020 . The enhanced biosafety secondary laboratory is particularly suitable for related operations that pose pathogenic microorganisms threats to humans or the environment. In addition, we have established a special radiology examination route to perform X-ray or CT examinations for highly suspected and confirmed patients, thus reducing the chance of crossinfection with other patients, and protecting the safety of medical staff. We used the ultrasound to evaluate the pulmonary disease, if necessary, so that we can reduce the risk of infection transmission among patients being transported.
Up to February 6, 2020, hundreds of medical staff were infected with 2019-nCoV. This is not only a huge challenge for medical resources, but also a huge psychological pressure on medical staff, so strict isolation and protection measures are top priority. Also, the epidemic period coincided with the Chinese New Year, and a large number of people returned to work from outside the hospital's region. Therefore, it is particularly important to conduct epidemiological screening of medical staff, especially enquiring about any Wuhan travel history and contact history with Wuhan people. We prepared a form and sent it to the WeChat group, asking each employee, as well as interns and residents, to report their epidemiological history. Once there was a high-risk or suspected 
person, strict isolation was required for 14 days. Training was carried out on hand hygiene, wearing isolation gowns, how to take nasopharyngeal and oropharyngeal swabs and environmental disinfection through online video or WeChat. We also introduced robotic systems to deliver meals and supplies to patients, reducing contact between medical staff and carriers, and reducing the chance of infection. We introduced a strictly control at the gates of the hospital, divided into the staff entrance and the patient entrance, and automatically monitor the temperature for everyone who enters the hospital.

The hospital allocates a large amount of funds to encourage scientific research on 2019-nCoV and encourages medical staff to design research about it. As ICU doctors, we designed a study on the efficacy of high-flow oxygen therapy and non-invasive ventilators in patients with mild to moderate respiratory failure of $2019-\mathrm{nCoV}$ pneumonia, and ECMO therapy in patients with severe respiratory failure, and other research on antiviral drugs and Chinese medicine for 2019-nCoV pneumonia. We hope we can bring some new hope to this sudden epidemic disease.

The sudden outbreak not only affected the mental health of medical staff and patients, but also affected their families and the general public, especially because the transmission route is not clear. According to statistics, there are more than 300,000 people from Wuhan in the Sichuan Province. Ordinary citizens are very afraid of this data, which generally causes symptoms of anxiety, irritability, and insomnia. As a large provincial hospital in western China, we have set up a psychological help phone line on February 6, 2020, with experts from mental health center who answer to various questions of psychological nature online $24 \mathrm{~h}$.

In conclusion, as an epidemic high contagious disease that threatens the global world, lots of things are not fully understood. It is a huge challenge for all hospitals and medical staff. At the moment, controlling the source of infection and reducing the spread of infection are the most important issues. I believe that with everyone's efforts, we will be able to defeat the virus.

\section{Author details \\ ${ }^{1}$ Department of Critical Care Medicine, Sichuan Academy of Medical Sciences and Sichuan Provincial People's Hospital, University of Electronic Science and Technology of China, Chengdu, People's Republic of China. ${ }^{2}$ Department of Hospital Administration, Sichuan Academy of Medical Sciences and Sichuan Provincial People's Hospital, University of Electronic Science and Technology of China, Chengdu, People's Republic of China. ${ }^{3}$ Department of Nephrol- ogy, Sichuan Academy of Medical Sciences and Sichuan Provincial People's Hospital, University of Electronic Science and Technology of China, Chengdu, People's Republic of China.}

\section{Acknowledgements}

We would like to thank all our hospital members for their efforts. They are hospital presidents Shaoping Deng. Xiaoqin Zhang, Yihui Zhang, Yiping Wang, Xiaoxiu Luo from Department of Critical Care Medicine, Sichuan Provincial People's Hospital. Head nurse Rong Lu, Qin Yang from Department of Critical Care Medicine, Sichuan Provincial People's Hospital. Infection control expert Jia Yu Wu, Qian Xiang from Department of Infectious Disease; Respiratory therapist Fuxun Yang from Department of Critical Care Medicine, Sichuan Provincial People's Hospital.

Funding

No.

\section{Compliance with ethical standards}

\section{Conflicts of interest}

On behalf of all authors, the corresponding author states that there is no conflict of interest.

\section{Publisher's Note}

Springer Nature remains neutral with regard to jurisdictional claims in published maps and institutional affiliations.

Received: 6 February 2020 Accepted: 8 February 2020

Published online: 18 February 2020

\section{Reference}

1. Huang CL, Wang Y, Li XW (2020) Clinical features of patients infected with 2019 novel coronavirus in Wuhan. Lancet, China. https://doi.org/10.1016/ S0140-6736(20)30183-5 